\title{
Smoking-related idiopathic interstitial pneumonia
}

\author{
Kevin R. Flaherty ${ }^{1,24}$, Charlene Fell2,24, Marie-Christine Aubry ${ }^{3}$, Kevin Brown ${ }^{4}$, \\ Thomas Colby 5 , Ulrich Costabel ${ }^{6}$, Teri J. Franks', Barry H. Gross ${ }^{8}$, \\ David M. Hansell', Ella Kazerooni ${ }^{8}$, Dong Soon Kim ${ }^{10}$, Talmadge E. King Jr ${ }^{11}$, \\ Masanori Kitachi ${ }^{12}$, David Lynch ${ }^{13}$, Jeff Myers ${ }^{14}$, Sonoko Nagai ${ }^{15}$, \\ Andrew G. Nicholson ${ }^{16}$, Venerino Poletti17, Ganesh Raghu ${ }^{18}$, Moises Selman ${ }^{19}$, \\ Galen Toews ${ }^{\dagger 1}$, William Travis ${ }^{20}$, Athol U. Wells ${ }^{21}$, Robert Vassallo ${ }^{22}$ and \\ Fernando J. Martinez ${ }^{1,23}$
}

\begin{abstract}
Affiliations: 'Division of Pulmonary and Critical Care Medicine, University of Michigan, Ann Arbor, MI, USA. ${ }^{2}$ Division of Respiratory Medicine, University of Calgary, Calgary, AB, Canada. ${ }^{3}$ Dept of Pathology, Mayo Clinic, Rochester, MN, USA. “Division of Pulmonary Medicine, National Jewish Medical and Research Center, Denver, CO, USA. ${ }^{5}$ Dept of Pathology, Mayo Clinic, Scottsdale, AZ, USA. ${ }^{6}$ Dept of Pneumology/Allergy, Ruhrlandklinik, University Hospital, Essen, Germany. ${ }^{7}$ Dept of Pulmonary and Mediastinal Pathology, The Joint Pathology Center, Silver Spring, MD, USA. ${ }^{8}$ Dept of Radiology, University of Michigan, Ann Arbor, MI, USA. ${ }^{9}$ Dept of Radiology, Royal Brompton Hospital, London, UK. ${ }^{10}$ Dept of Pulmonary and Critical Care Medicine, Asan Medical Center, University of Ulsan, Seoul, Korea. ${ }^{11}$ Division of Pulmonary, Critical Care, Allergy and Sleep Medicine, University of California, San Francisco, CA, USA. ${ }^{12}$ National Hospital Organization Kinki-Chuo, Osaka, Japan. ${ }^{13}$ Dept of Radiology, National Jewish Medical and Research Center, Denver, CO, USA. ${ }^{14}$ Dept of Pathology, University of Michigan, Ann Arbor, MI, USA. ${ }^{15}$ Respiratory Medicine, Kyoto University, Kyoto, Japan. ${ }^{16}$ Dept of Histopathology, Royal Brompton Hospital, London, UK. ${ }^{17}$ Dipartimento di Malattie del Torace, Universita di Parma, Forli, Italy. ${ }^{18}$ Division of Pulmonary Medicine, University of Washington, Seattle, WA, USA. ${ }^{19}$ Instituto Nacional de Enfermedades Respiratorias, Mexico DF, Mexico. ${ }^{20}$ Dept of Pathology, Memorial Sloan Kettering Cancer Center, New York, NY, USA. ${ }^{21}$ Interstitial Lung Disease Unit, Royal Brompton Hospital, London, UK. ${ }^{22}$ Division of Pulmonary, Allergy and Critical Care Medicine, Mayo Clinic, Rochester, MN, USA. ${ }^{23}$ Dept of Medicine, Division of Pulmonary and Critical Care Medicine, Weill Cornell Medical Center, New York, NY, USA. ${ }^{24}$ Both authors contributed equally.
\end{abstract}

Correspondence: Fernando J. Martinez, Dept of Internal Medicine, Division of Pulmonary and Critical Care Medicine, Weill Cornell Medical School, New York, NY, USA. E-mail: fjm2003Amed.cornell.edu

ABSTRACT Cigarette smoking is a key factor in the development of numerous pulmonary diseases.

An international group of clinicians, radiologists and pathologists evaluated patients with previously identified idiopathic interstitial pneumonia (IIP) to determine unique features of cigarette smoking. Phase 1 (derivation group) identified smoking-related features in patients with a history of smoking $(\mathrm{n}=41)$. Phase 2 (validation group) determined if these features correctly predicted the smoking status of IIP patients $(n=100)$ to participants blinded to smoking history. Finally, the investigators sought to determine if a new smoking-related interstitial lung disease phenotype could be defined.

Phase 1 suggested that preserved forced vital capacity with disproportionately reduced diffusing capacity of the lung for carbon monoxide, and various radiographic and histopathological findings were smoking-related features. In phase 2, the kappa coefficient among clinicians was 0.16 (95\% CI 0.11-0.21), among the pathologists 0.36 (95\% CI 0.32-0.40) and among the radiologists 0.43 (95\% CI 0.35-0.52) for smoking-related features. Eight of the 100 cases were felt to represent a potential smoking-related interstitial lung disease.

Smoking-related features of interstitial lung disease were identified in a minority of smokers and were not specific for smoking. This study is limited by its retrospective design, the potential for recall bias in smoking history and lack of information on second-hand smoke exposure. Further research is needed to understand the relationship between smoking and interstitial lung disease.

@ERSpublications

Associations between smoking and ILD are largely nonspecific, but a small group may have smoking-related ILD http://ow.ly/wExAf

The content of this work is not subject to copyright. Design and branding are @ERS 2014. 


\section{Introduction}

Cigarette smoking is the leading cause of chronic obstructive pulmonary disease in developed countries [1]. Smoking is also associated with diffuse parenchymal lung diseases such as respiratory bronchiolitis interstitial lung disease (RB-ILD), desquamative interstitial pneumonia (DIP), idiopathic pulmonary fibrosis (IPF) and pulmonary Langerhans' cell histiocytosis (PLCH) [2-6]. Growing interest has developed around the idea that there may be a smoking-related interstitial lung disease (ILD) phenotype. In particular, combined lower lobe pulmonary fibrosis and upper lobe emphysema has been emphasised as a distinct entity [7-17] and smoking-related interstitial fibrosis may be associated with a specific histopathological pattern $[18,19]$.

This study was performed by an international group of pulmonary physicians, radiologists and pathologists who retrospectively evaluated the clinical history, radiographic and histopathological materials from patients initially presenting with suspected idiopathic interstitial pneumonias (IIPs). An initial derivation phase sought to identify smoking-related features from patients with a history of smoking. A second validation phase sought to determine if investigators could use these features to correctly predict the smoking status of patients (when the smoking history was withheld) and, thus, provide at least indirect evidence that unique smoking-related features are present in patients with IIP. Finally, investigators sought to determine if a new smoking-related ILD phenotype could be defined. This study is limited by its retrospective design, the potential for recall bias in smoking history and lack of information on secondhand smoke exposure.

\section{Methods \\ Organisation of the expert panel}

The primary goal of this project was to define the clinical, radiological and pathological features of smoking-related interstitial pneumonia based on a pooled dataset of cases with clinical, chest highresolution computed tomography (HRCT) and surgical lung biopsy (SLB) data. To develop a broad consensus on this complicated topic, an international panel of expert clinicians, radiologists and pathologists was organised (from Canada, Germany, Italy, Japan, Korea, Mexico, the UK and the USA). This project was organised in two phases. Phase 1 consisted of an initial review of selected cases with a history of smoking to determine a consensus for specific smoking-related features. In phase 2 we employed the criteria developed in phase 1 to determine if the criteria allowed for the identification of cases with a history of smoking. Not all experts participated in both phases (see later). This project was sponsored by the American Thoracic Society, the European Respiratory Society, the Japanese Respiratory Society and the Korean Academy of Tuberculosis and Respiratory Diseases. Institutional review board approval was granted to review the case material for this study.

\section{Collection of the cases and data reviewed}

A total of 141 cases of IIP were reviewed during the course of this study. This project was organised in two phases. The phase 1 derivation stage consisted of initial review of selected cases $(n=41)$ with a history of smoking to determine a consensus for specific smoking-related features. In phase 2, the validation stage $(n=100)$, we employed the criteria developed in phase 1 to determine if the criteria allowed for the identification of cases with a history of smoking. For the purposes of this study, a smoker was considered as any subject with any history of smoking. Former smokers were subjects that had a history of smoking, but had quit prior to SLB. Not all experts participated in both phases (see later).

In each phase of the study, available clinical data included age, presence and duration of symptoms (cough, dyspnoea, fever, weight loss, myalgias, arthritis, rash or Raynaud's), physical examination findings (crackles, clubbing or other), occupational/hobby exposures, pulmonary function, arterial blood gas, and serological test results. A HRCT study within 6 months of a SLB was available in all patients.

This article has supplementary material available from erj.ersjournals.com

Disclaimer (T.J. Franks): the views expressed in this article are those of the author and do not reflect the official policy of the Department of Defense or the United States Government.

Received: Sept 232013 | Accepted after revision: April 222014 | First published online: July 252014

Support statement: This study was supported by the grants from the American Thoracic Society, European Respiratory Society, Japanese Respiratory Society, Korean Academy of Tuberculosis and Respiratory Diseases, and the Alberta Heritage Foundation for Medical Research.

Conflict of interest: Disclosures can be found alongside the online version of this article at erj.ersjournals.com 
Phase 1: derivation group

41 cases of IIP with a known positive smoking history were selected by investigators from the USA, UK, Mexico and Korea. The groups initially worked independently with clinicians $(n=8)$ reviewing clinical data and HRCTs, radiologists $(n=3)$ reviewing HRCTs, and pathologists $(n=6)$ reviewing histopathology. Clinicians initially recorded diagnostic impressions for each case as well as comments about the clinical and HRCT features present and the possibility of certain features being attributable to smoking. Radiologists and pathologists used a score sheet to note the presence/absence of pre-specified features as well as a graded impression of smoking relatedness. Radiologists scored the presence/absence of honeycombing, reticular thickening, cysts, nodules, ground-glass opacities, consolidation, emphysema, mosaic attenuation, disease distribution and other features as well as the overall diagnostic impression. Pathologists scored the presence/ absence of emphysema, emphysema-like airspace enlargement with fibrosis, acellular hyaline fibrosis of alveolar walls, pigmented macrophages, respiratory bronchiolitis, DIP-like foci, bronchiolocentric stellate scarring and other features, as well as overall diagnostic impression. Radiologists utilised a three-tier system (0: none; 1 : mild; 2 : definitely related to smoking) while pathologists used a five-tier system (1: definite; 2: probable; 3: possible; 4: definitely not; 5 : do not know if related to smoking).

\section{Phase 2: validation group}

In the second phase of this study, five expert clinicians, three thoracic radiologists and five pulmonary pathologists individually reviewed 100 cases of IIP (48 never-smokers and 52 former/current smokers) to determine the diagnosis, smoking status and contribution of smoking-related changes (if any) to the primary pathological process. We also attempted to determine if a unique smoking-related ILD existed and could be culled from the current IIP classifications. Some, but not all, physicians had participated in phase 1 of the study. All physicians were blinded to the smoking history of the patients. Cases were selected from the UK, Germany, Korea, Japan, Mexico and the USA. Each individual classified the features present and utilised the presence or absence of features to predict the patients' smoking status.

Following individual review, all physicians collectively reviewed the 100 cases and determined if any met the predefined criteria from phase 1 for smoking-related ILD. A consensus diagnosis of "smoking-related ILD”, "ILD in a smoker" or "ILD in a nonsmoker" was assigned to each case. Assignment to each group was made by general consensus using features in table 1; a specific definition for each group was not specified. A subject was considered to be a probable smoker if the majority of clinicians (three out of five), radiologists (two out of three) or pathologists (three out of five) classified that subject as a smoker.

\section{Statistical analysis}

Descriptive statistics were used to characterise the demographic characteristics for subjects in phase 1 and 2. Differences between groups were tested with t-test and Chi-squared statistics. Kappa coefficients of interrater agreement were determined for each type of physician $(n=500$ observations each for the clinicians and pathologists, and $n=300$ observations for the radiologists). Data are expressed as mean \pm SD or frequency (\%). Tests were significant when $\mathrm{p}<0.05$. Statistical analysis was performed with SPSS 14.0 (SPSS Inc., Chicago, IL, USA) and SAS 9.1 (SAS Institute Inc., Cary, NC, USA) software packages.

\section{Results}

A total of 141 cases of IIP were reviewed during the course of this study. 41 subjects were evaluated in the phase 1 definition stage and 100 subjects in the phase 2 validation stage.

\section{Phase 1}

Demographic characteristics of the cases are shown in table 2. All of the cases had a history of smoking. There were no differences in age, sex or clinical features between the former and current smokers. Lung function was also similar between the two groups; however, former smokers had a lower diffusing capacity of the lung for carbon monoxide (DLCO) \% predicted compared with current smokers. Pathological patterns included usual interstitial pneumonia (UIP; $n=10$ ), nonspecific interstitial pneumonia (NSIP; $n=11)$, RB-ILD/DIP $(n=14)$, cryptogenic organising pneumonia (COP; $n=4)$ and non-classifiable $(n=3)$.

Clinical, radiographic and histopathological features that were felt, by consensus discussion during case review, to be attributable to smoking and thus representative of a smoking-related ILD phenotype were identified (table 1). These included preserved pulmonary mechanics with disproportionately reduced DLCO, radiographic evidence of emphysema, centrilobular nodules, cysts in ground-glass opacity (fig. 1a and b) and histopathological features of emphysema, emphysema-like airspace enlargement, respiratory bronchiolitis, DIP-like foci, and bronchiolocentric stellate scars, i.e. a lesion suggestive of healed PLCH lesions (fig. 2a-c). 
TABLE 1 Clinical, radiographic and histopathological features attributed to smoking in patients with idiopathic interstitial pneumonia

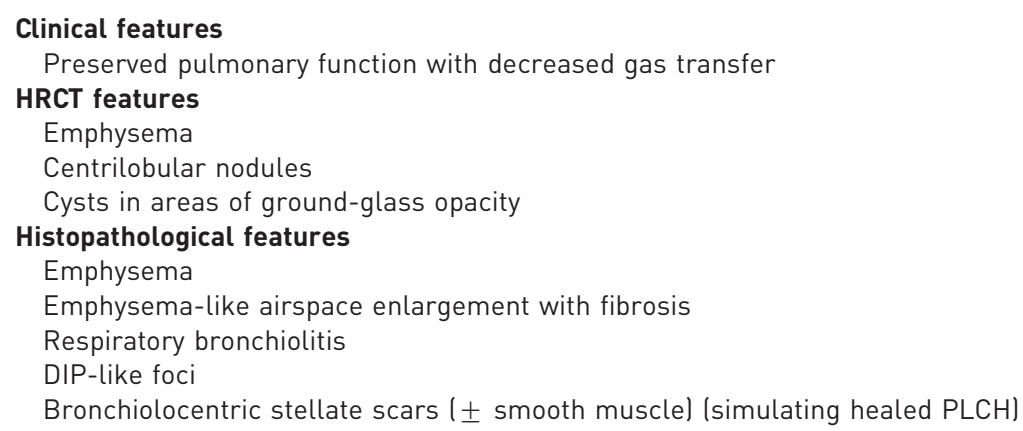

HRCT: high-resolution computed tomography; DIP: desquamative interstitial pneumonia; PLCH: pulmonary Langerhans' cell histiocytosis.

\section{Phase 2}

Demographic and known clinical characteristics of the patients $(n=100 ; 48$ never-smokers and 52 eversmokers) are outlined in table 3. Never-smokers were younger, more likely to be female and had lower forced vital capacity (FVC) \% pred than current or former smokers. Pathological patterns included UIP $(\mathrm{n}=44)$, NSIP $(\mathrm{n}=21)$, RB-ILD/DIP $(\mathrm{n}=18)$, hypersensitivity pneumonia $(\mathrm{n}=5)$, end-stage lung disease $(n=4)$, acute lung injury $(n=1), \operatorname{COP}(n=1)$ and pulmonary venous occlusive disease $(n=1)$. A consensus pathological pattern was not reached in four cases.

The ability of participants to agree on the presence of smoking-related features determined from phase 1 (table 1) was fair to moderate as assessed by the kappa coefficient. The kappa coefficient among clinicians was 0.16 (95\% CI $0.11-0.21$ ), among the pathologists 0.36 (95\% CI $0.32-0.40)$ and among the radiologists 0.43 (95\% CI 0.35-0.52). Furthermore, the overall ability of participants to correctly classify subjects as smokers/non-smokers utilising features from phase 1 was poor (table 4).

All members of the panels met collectively to review the 100 cases analysed in phase 2 and determine if any met the predefined criteria from phase 1 for smoking-related ILD (table 5). Eight of the 100 cases were felt to represent a potential smoking-related ILD, 13 cases were deemed ILD in smokers (patients with clinical, radiographic and/or histopathological evidence of smoking) and 79 cases were not otherwise reclassified (table 5). Cases reclassified as a potential smoking-related ILD were predominantly of British origin and all

TABLE 2 Demographic and physiological data from 41 cases of smokers with idiopathic interstitial pneumonia from phase 1 (derivation group)

\begin{tabular}{|c|c|c|c|}
\hline & Former smokers & Current smokers & p-value \\
\hline Subjects & 14 & 27 & \\
\hline Age years & $55.1 \pm 8.7$ & $52.7 \pm 11.2$ & 0.49 \\
\hline Males/females & $11 / 3$ & $20 / 7$ & 0.23 \\
\hline Smoking history pack-years & $34.5 \pm 24.59$ & $38.4 \pm 19.3$ & 0.59 \\
\hline \multicolumn{4}{|l|}{ Clinical history } \\
\hline Cough yes/no & $13 / 1$ & $24 / 2$ & 0.96 \\
\hline Duration of cough months & $18.5 \pm 18.3$ & $12.4 \pm 16.7$ & 0.30 \\
\hline Dyspnoea yes/no & $14 / 0$ & $26 / 1$ & 0.75 \\
\hline Duration of dyspnoea months & $18.9 \pm 17.0$ & $13.1 \pm 17.2$ & 0.31 \\
\hline \multicolumn{4}{|l|}{ Pulmonary function } \\
\hline FVC \% predicted & $71.7 \pm 22.6$ & $77.0 \pm 19.3$ & 0.44 \\
\hline FEV1 $\%$ predicted & $76.4 \pm 18.2$ & $75.5 \pm 18.8$ & 0.89 \\
\hline $\mathrm{FEV} 1 / \mathrm{FVC}$ & $81.3 \pm 8.9$ & $77.1 \pm 12.5$ & 0.28 \\
\hline DLCO \% predicted & $46.1 \pm 18.1$ & $57.0 \pm 12.5$ & 0.03 \\
\hline
\end{tabular}


a)

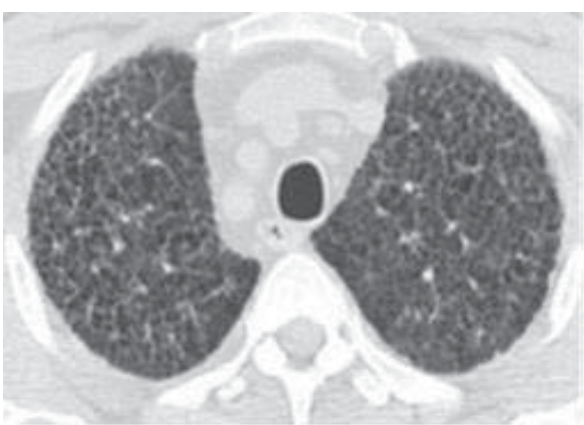

b)

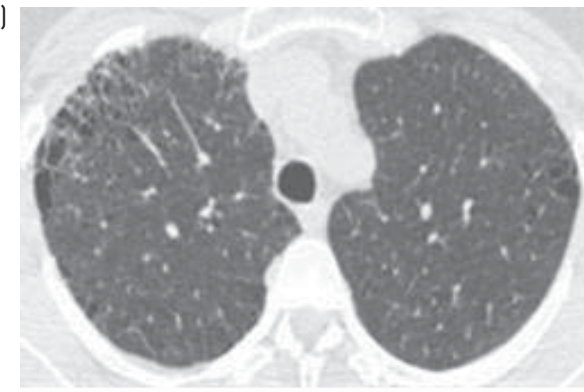

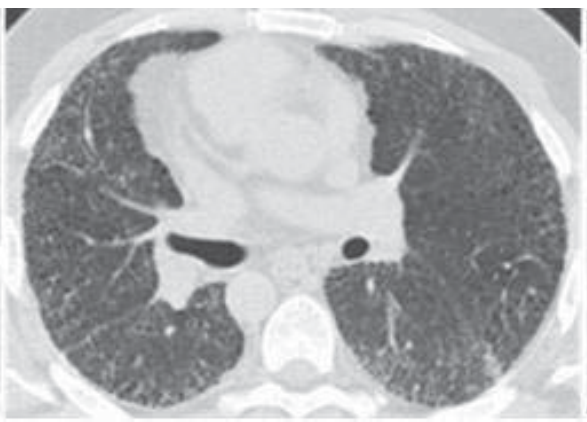

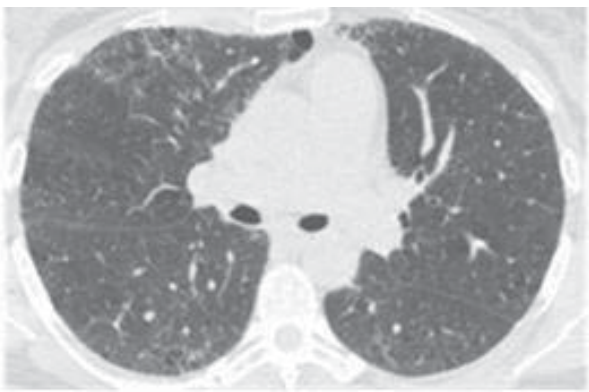

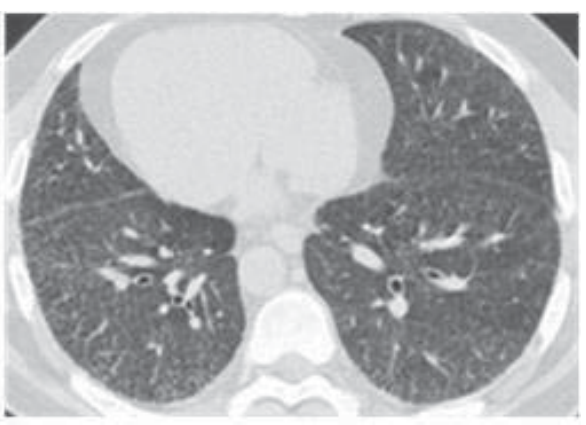

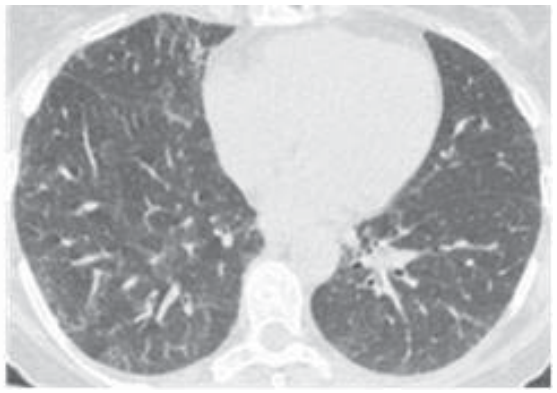

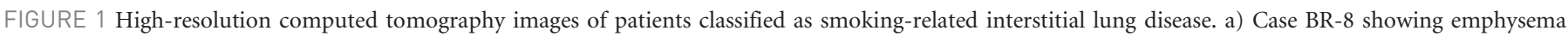
and reticulation; b) Case BR-11 showing cysts in areas of ground-glass opacification.

had a numerically greater pack-year history of smoking than cases reclassified as ILD in a smoker or other. Interestingly, one case classified as smoking-related ILD was a never-smoker, which, even retrospectively, was felt to represent an inaccurate history by the study group. There were no significant differences in overall symptoms. The group of subjects classified as smoking-related ILD had a more preserved FVC compared with other groups, although other measures of lung function were similar (table 5).

\section{Discussion}

Smoking, either directly or through second-hand smoke exposure, has been implicated in numerous pulmonary diseases including the diffuse parenchymal lung disorders. The histopathological features attributed to cigarette smoke may exist across specific diagnostic disorders and can be seen in cases that fail to fit into currently described diagnostic patterns. Although abnormalities attributed to cigarette smoking are common, a distinct smoking-related ILD phenotype has not been defined. This study tested the hypothesis that changes attributed to smoking can be identified in patients with suspected IIP and be used to predict a history of smoking. We also explored if a unique smoking-related ILD phenotype could be culled from the larger group of IIPs. Data from this study document indicate that although distinct clinical, radiological and pathological findings were felt to suggest an association between smoking and ILD, these features were diverse and nonspecific and could not be reliably used by physicians to accurately identify if cases under review truly had a history of smoking. A smoking-related ILD phenotype may be present; however, it is found in a minority of ILD cases with documented smoking history. A smoking-related ILD phenotype may be sufficiently uncommon (no more than $8 \%$ of IIP) that our case numbers were too small to confidently identify and characterise such a subgroup.

Features attributed to smoking in this study extend previously published descriptions of smoking-related features of ILD. Clinicians in this study identified relatively preserved pulmonary mechanics with disproportionately reduced DLCO as suggestive of smoking-related ILD. This supports previously published data regarding the superimposition of emphysema and IPF [20-22]. Radiologists identified emphysema, centrilobular nodules, respiratory bronchiolitis and changes suggestive of NSIP (ground-glass attenuation with or without intrapulmonary cystic areas). These findings are similar to those reported in other settings $[23,24]$. Pathologists identified emphysema and emphysema-like airspace enlargement with fibrosis, respiratory bronchiolitis, DIP-like foci and bronchiolocentric stellate scars indicative of healed PLCH lesions as suggestive of smoking-related ILD [19, 23, 25]. These findings are not all-inclusive for changes that could occur with cigarette smoking, but seemed to best fit as smoking-related cases when cases with and without a history of smoking were reviewed knowing a priori the smoking history. 

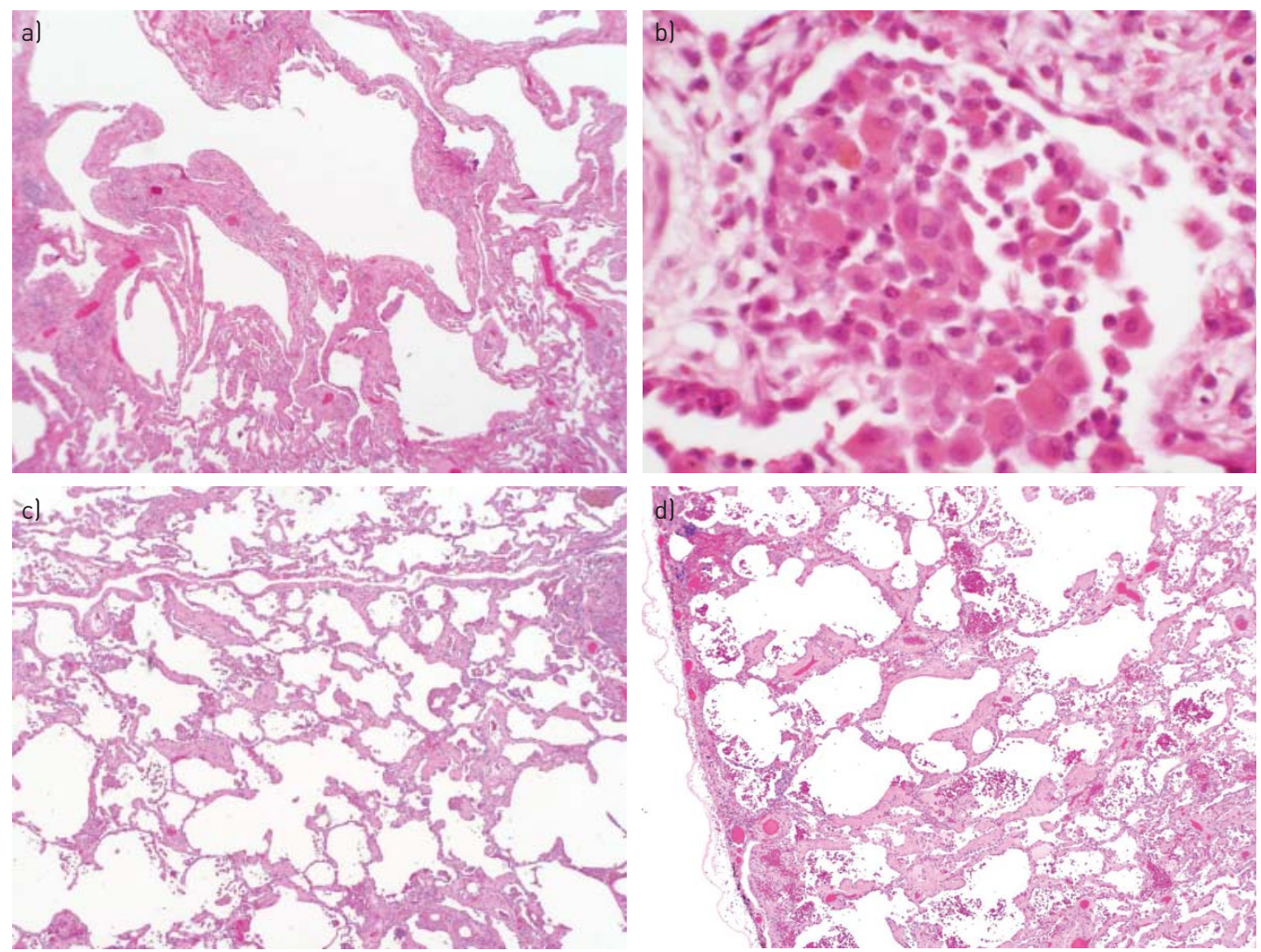

FIGURE 2 Histopathological features associated with smoking in patients with interstitial lung disease (ILD). a) Fibrosis and emphysema in a patient with usual interstitial pneumonia (original magnification: $100 \times$ ). b) Respiratory bronchiolitis-ILD changes in a patient with nonspecific interstitial pneumonia (NSIP) (original magnification: $400 \times$ ). c) and d) Respiratory bronchiolitis versus NSIP in a smoker (original magnification: $40 \times$ ).

Although several clinical, radiographic and histopathological features were felt to represent changes attributable to smoking, the identification of these features failed to accurately represent the true smoking history in a significant number of subjects. There was significant disagreement between the true smoking history and the assignment of smoking history by all observers. Features attributable to smoking were seen

TABLE 3 Demographic and physiological data for 100 cases of idiopathic interstitial pneumonia presented in phase 2 (validation group)

\section{Never-smokers}

Current/former smokers

p-value

\begin{tabular}{lccc}
\hline Subjects & 48 & 52 & \\
Age years & $53.0 \pm 10.7$ & $55.6 \pm 12.2$ & 0.259 \\
Males/females/NA & $18 / 27 / 3$ & $33 / 18 / 1$ & 0.013 \\
Smoking history pack-years & 0 & $31.4 \pm 32.8$ & $<0.005$ \\
Clinical history & & & \\
$\quad$ Cough yes/no/NA & $38 / 6 / 4$ & $37 / 14 / 1$ & 0.100 \\
$\quad$ Duration of cough months & $28.2 \pm 42.3$ & $22.4 \pm 27.0$ & 0.479 \\
Dyspnoea yes/no & $46 / 2$ & $47 / 5$ & 0.286 \\
Duration of dyspnoea months & $21.2 \pm 35.3$ & $27.2 \pm 31.2$ & 0.399 \\
Pulmonary function & & & \\
FVC \% predicted & $61.9 \pm 15.9$ & $70.5 \pm 21.9$ & 0.028 \\
FEV1 \% predicted & $68.5 \pm 16.3$ & $74.7 \pm 21.3$ & 0.106 \\
FEV1/FVC & $96.7 \pm 16.4$ & $89.6 \pm 17.2$ & 0.043 \\
TLC \% predicted & $67.0 \pm 14.1$ & $75.8 \pm 17.6$ & 0.01 \\
DLCO \% predicted & $48.9 \pm 18.2$ & $50.4 \pm 19.8$ & 0.711 \\
\hline
\end{tabular}

Data are presented as $n$ or mean \pm SD, unless otherwise stated. NA: not available; FVC: forced vital capacity; FEV1: forced expiratory volume in $1 \mathrm{~s}$; TLC: total lung capacity; DLCO: diffusing capacity of the lung for carbon monoxide. 
TABLE 4 Classification of patients with idiopathic interstitial pneumonia as ever-smokers and never-smokers stratified by actual history of smoking

\begin{tabular}{|c|c|c|c|c|}
\hline & Clinicians & Radiologists & Pathologists & $\begin{array}{c}\text { Chi-squared } \\
\text { p-value }\end{array}$ \\
\hline Never-smokers ${ }^{\#}$ & & & & 0.008 \\
\hline Not a smoker & 27 & 40 & 37 & \\
\hline A smoker & 21 & 8 & 11 & \\
\hline Current smokers ${ }^{\pi}$ & & & & 0.92 \\
\hline Not a smoker & 6 & 6 & 5 & \\
\hline A smoker & 14 & 14 & 15 & \\
\hline Former smokers ${ }^{+}$ & & & & 0.037 \\
\hline Not a smoker & 11 & 21 & 14 & \\
\hline A smoker & 21 & 11 & 18 & \\
\hline
\end{tabular}

Data are presented as $n$, unless otherwise stated. ${ }^{\#}: n=48 ;{ }^{\natural}: n=20 ;{ }^{+}: n=32$.

in nonsmokers and patients with a history of smoking often lacked "attributable" features of smoking. This was particularly evident in the assignment by clinicians, but also applied to radiologists who used a broad spectrum of features including the presence of emphysema, interlobular septal thickening and centrilobular nodules [23]. Pathologists also used a broad range of features including the presence of emphysema, acellular hyaline fibrosis, respiratory bronchiolitis, pigmented macrophages, DIP foci, bronchiolocentric scars, and the presence and distribution of fibroblastic foci $[19,23]$. Two investigative groups have recently documented the frequent finding of smoking-related fibrosis in patients undergoing lobectomy for presumed malignancy $[18,19,26]$. A correlation between smoking history and airspace enlargement with fibrosis was suggested by one of these groups [19], while the other group felt the findings suggested a specific smoking-related pattern of fibrosis [18]. Importantly, in our group of subjects, "smoking-related" pathological changes were identified in some nonsmokers. This may relate to a common mechanism of injury and repair, as opposed to a common toxicological form of injury. For example, respiratory bronchiolitis and RB-ILD are seen in smokers, but DIP may be seen in both smokers and nonsmokers (due to drugs, infection or other insults). In respiratory bronchiolitis and RB-ILD cigarette smoke is the common injury, whereas DIP may represent manifestations of a common injury/repair mechanism. It is also possible

TABLE 5 Demographic and clinical features of cases labelled by study participants as smoking-related interstitial lung disease (ILD), ILD in a smoker or other

Smoking-related ILD

ILD in a smoker

Other

p-value

Subjects
Age years
Males/females/NA
Smoking history
Never
Former/current
Pack-years
Clinical history
Cough yes/no/NA
Cough duration months
Dyspnoea yes/no
Dyspnoea duration months
Pulmonary function
FVC \% predicted
FEV $1 \%$ predicted
FEV1/FVC
TLC \% predicted
DLCO \% predicted

$\begin{array}{cc}8 & 13 \\ 47.00 \pm 5.00 & 54.64 \pm 10.93 \\ 4 / 3 / 1 & 5 / 8 \\ & \\ 1 & 6 \\ 7 & 7 \\ 34.17 \pm 29.90 & 17.83 \pm 22.69 \\ 5 / 1 / 2 & 9 / 4 / 0 \\ 16.2 \pm 11.14 & 31.00 \pm 41.53 \\ 8 / 0 & 13 / 0 \\ 20.62 \pm 16.31 & 31.08 \pm 39.46 \\ & \\ 81.90 \pm 19.09 & 69.38 \pm 9.05 \\ 79.75 \pm 18.28 & 77.38 \pm 10.94 \\ 74.50 \pm 9.93 & 93.50 \pm 14.50 \\ 91.17 \pm 10.98 & 74.92 \pm 10.59 \\ 48.87 \pm 13.72 & 46.08 \pm 19.28\end{array}$

\section{9}

$54.98 \pm 11.82$

$42 / 34$

0.211

0.520

0.103

41

38
$3.73 \pm 28.19 \quad 0.215$

0.642

$61 / 15 / 3$

$\begin{array}{ll}24.80 \pm 35.88 & 0.737\end{array}$

$72 / 7 \quad 0.368$

$23.48 \pm 33.76 \quad 0.732$

$64.33 \pm 20.38 \quad 0.045$

$69.90 \pm 20.15 \quad 0.202$

$94.33 \pm 17.17 \quad 0.02$

$68.9 \pm 16.67 \quad 0.0039$

$50.39 \pm 19.47 \quad 0.750$

Data are presented as $n$ or mean \pm SD, unless otherwise stated. NA: not available; FVC: forced vital capacity; FEV1: forced expiratory volume in $1 \mathrm{~s}$; TLC: total lung capacity; DLCO: diffusing capacity of the lung for carbon monoxide. 
that changes in our nonsmoking subjects that were attributed to smoking could relate to second-hand smoke or pollution, factors that could not be verified in our subjects.

At consensus review of the validation cohort only eight of the 100 cases were felt to represent a smokingrelated ILD phenotype utilising features from phase 1, with an additional 13 cases showing strong evidence for smoking in addition to underlying ILD. The majority of patients in the second phase cohort had IPF as the final diagnosis. Those patients with a possible smoking-related ILD were more likely to be from the UK, but otherwise exhibited similar demographic, clinical and physiological features to the other patient groups. One patient classified in the smoking-related ILD group was a nonsmoker. There are numerous possibilities for these discrepancies. The first is related to the retrospective nature of the study. The possibility for misclassification of smoking status either through deception or recall bias is clearly possible. In addition, we did not have access to detailed information about second-hand smoke or levels of environmental pollution so it is possible that some of our nonsmoking subjects actually had significant smoke exposure. Second, the definition of smoking-related ILD features from phase 1 were purposefully restrictive in the hope of being specific. We tended to identify the patients with emphysema and coexistent ILD. There is clearly the potential for other manifestations of smoking injury including patients with more restrictive or mixed obstructive/restrictive physiology, diffuse ground-glass opacity on HRCT, or overlaps between cysts/ emphysema/ground-glass opacity. It is also possible that features attributed to smoking, such as centrilobular nodules and respiratory bronchiolitis, may be recognised in patients without smokingrelated lung injury. It is well established that similar histopathological patterns (such as UIP, organising pneumonia, etc.) can be seen in response to numerous types of injury or diseases and, thus, histopathological patterns are rarely, if ever, specific for a disease without clinical and pathological correlation [27]. Finally, an individual patient's response to injury is likely to be different to responses identified in a population; in large groups of smokers, it is possible to identify patients with normal lung function, emphysema and chronic bronchitis. Thus, the possibility for a differential response to injury clearly exists.

Strengths of this study include the large number of well-characterised patient samples evaluated, as well as the participation of a large group of international experts with a broad range of experience. The diverse patient population is also considered a strength although different environmental factors, practice patterns (when to carry out pulmonary function tests, HRCT or biopsy) or genetic/racial responses to injury could influence the smoking-ILD relationship. The inclusion of derivation and validation groups with blinding of the participants during the second phase also adds strengths to the observations. The lack of a prospectively assigned treatment regimen and long-term follow-up are weaknesses. Another potential weakness is the study population size if a putative smoking-related ILD is relatively rare (and would, thus, require an even larger study sample to confidently identify).

In summary, a large international cohort of clinicians, radiologists and pathologists with expertise in ILD retrospectively reviewed a collection of clinical, radiographic and histopathological data to establish if specific smoking-related features of ILD and a specific smoking-related ILD could be identified. These features were identified in a minority of heavy smokers; however, these features were absent in some patients with a history of smoking and present in some patients without a history of smoking. The finding that one of the eight patients regarded by all specialists to have smoking-related ILD was a never-smoker without passive smoke exposure suggests that other causes exist for this phenotype. Thus, smoking is strongly associated with ILD, but it is difficult to distinguish smoking-related ILD from non-smokingrelated ILD unless the smoking history is available to the clinician. Further research is needed to better understand the clinical manifestations of smoking and ILD as well as implications for treatment and prognosis.

\section{Acknowledgements}

The authors gratefully thank Ken Guire (Dept of Biostatistics, School of Public Health, University of Michigan, Ann Arbor, MI, USA) for his assistance analysing data for this project. In addition, the authors wish to gratefully thank Gary Hunninghake, Roland du Bois and Andrew Flint for their aid during the data collection phase of the study.

\section{References}

1 Fromer L, Cooper C. A review of the gold guidelines for the diagnosis and treatment of patients with COPD. Int J Clin Pract 2008; 62: 1219-1236.

2 American Thoracic Society, European Respiratory Society. American Thoracic Society/European Respiratory Society international multidisciplinary consensus classification of the idiopathic interstitial pneumonias. Am J Respir Crit Care Med 2002; 165: 277-304.

3 Ryu JH, Colby TV, Hartman T, et al. Smoking-related interstitial lung diseases: a concise review. Eur Respir J 2001; 17: $122-132$.

4 Ryu JH, Myers JL, Capizzi SA, et al. Desquamative interstitial pneumonia and respiratory bronchiolitis-associated interstitial lung disease. Chest 2005; 127: 178-184. 
Steele MP, Speer MC, Loyd JE, et al. Clinical and pathologic features of familial interstitial pneumonia. Am J Respir Crit Care Med 2005; 172: 1146-1152.

Vassallo R, Ryu JH. Smoking-related interstitial lung diseases. Clin Chest Med 2012; 33: 165-178

Cottin V, Cordier J. The syndrome of combined pulmonary fibrosis and emphysema. Chest 2009; 136 : 1-2.

Hiwatari N, Shimura S, Takishima T. Pulmonary emphysema followed by pulmonary fibrosis of undetermined cause. Respiration 1993; 60: 354-358.

9 Millar AB, Denison DM. Vertical gradients of lung density in supine subjects with fibrosing alveolitis or pulmonary emphysema. Thorax 1990; 45: 602-605.

10 Wiggins J, Strickland B, Turner-Warwick M. Combined cryptogenic fibrosing alveolitis and emphysema: The value of high resolution computed tomography in assessment. Respir Med 1990; 1990: 365-369.

11 Mejía M, Carrillo G, Rojas-Serrano J, et al. Idiopathic pulmonary fibrosis and emphysema: decreased survival associated with severe pulmonary arterial hypertension. Chest 2009; 136: 10-15.

12 Oliva IB, Cortopassi F, Rochester CL, et al. Combined pulmonary fibrosis and emphysema syndrome: a radiologic perspective. Monaldi Arch Chest Dis 2011; 75: 220-234.

13 Portillo K, Morera J. Combined pulmonary fibrosis and emphysema syndrome: a new phenotype within the spectrum of smoking-related interstitial lung disease. Pulm Med 2012; 2012: 867870.

14 Jankowich MD, Rounds SI. Combined pulmonary fibrosis and emphysema syndrome: a review. Chest 2012; 141: $222-231$.

15 Samara KD, Magaritopoulos G, Wells AU, et al. Smoking and pulmonary fibrosis; novel insights. Pulm Med 2011; 2011: 461439 .

16 Lee $\mathrm{CH}$, Kim HJ, Park CM, et al. The impact of combined pulmonary fibrosis and emphysema on mortality. Int J Tuberc Lung Dis 2011; 15: 1111-1116.

17 Todd NW, Jeudy J, Lavania S, et al. Centrilobular emphysema combined with pulmonary fibrosis results in improved survival. Fibrogenesis Tissue Repair 2011; 4: 6.

18 Katzenstein AL, Mukhopadhyay S, Zanardi C, et al. Clinically occult interstitial fibrosis in smokers: classification and significance of a surprisingly common finding in lobectomy specimens. Hum Pathol 2010; 41: 316-325.

19 Kawabata Y, Hoshi E, Murai K, et al. Smoking-related changes in the background lung of specimens resected for lung cancer: a semiquantitative study with correlation to postoperative course. Histopathology 2008; 53: 707-714.

20 Cherniack RM, Colby TV, Flint A, et al. Correlation of structure and function in idiopathic pulmonary fibrosis. Am J Respir Crit Care Med 1995; 151: 1180-1188.

21 Cottin V, Nunes H, Brillet PY, et al. Combined pulmonary fibrosis and emphysema: a distinct underrecognised entity. Eur Respir J 2005; 26: 586-593.

22 Doherty MJ, Pearson MG, O’Grady EA, et al. Cryptogenic fibrosing alveolitis with preserved lung volumes. Thorax 1997; 52: 998-1002.

23 Attili AK, Kazerooni EA, Gross BH, et al. Smoking-related interstitial lung disease: radiologic-clinical-pathologic correlation. Radiographics 2008; 28: 1383-1398.

24 Lederer DJ, Enright PL, Kawut SM, et al. Cigarette smoking is associated with subclinical parenchymal lung disease: the Multi-Ethnic Study of Atherosclerosis (MESA)-lung study. Am J Respir Crit Care Med 2009; 180: 407-414.

25 Vassallo R, Ryu JH. Tobacco smoke-related diffuse lung diseases. Semin Respir Crit Care Med 2008; 29: 643-650.

26 Yousem SA. Respiratory bronchiolitis-associated interstitial lung disease with fibrosis is a lesion distinct from fibrotic nonspecific interstitial pneumonia: a proposal. Mod Pathol 2006; 19: 1474-1479.

27 Maher TM, Wells AU, Laurent GJ. Idiopathic pulmonary fibrosis: multiple causes and multiple mechanisms? Eur Respir J 2007; 30: 835-839. 